\title{
Innate Inflammatory Reaction of the Brain in Alzheimer Disease
}

\author{
Edith G. McGeer*, Ph.D., and Patrick L. McGeer, M.D.
}

\section{INTRODUCTION}

Evidence obtained over the past decade indicates that the brain has its own innate immune system which can sustain a low grade, chronic inflammatory reaction (reviewed in (1)). Such a chronic inflammatory reaction is silent because the brain lacks pain fibers, temperature changes cannot be measured, and the blood brain barrier largely isolates the brain from the blood borne elements responsible for the swelling which typically accompanies inflammation in peripheral tissues.

Presumably, the innate immune system of brain, like that of other tissues, is designed as a defense mechanism - to respond immediately to foreign invaders and to clean up debris from any type of injury. But the resultant inflammatory process may damage healthy tissue. The brain, instead of being immunologically privileged, may be particularly vulnerable to such processes since neurons are postmitotic and, once lost, cannot be replaced.

There is excellent evidence that inflammatory processes may be a secondary, but very important, mechanism of neuronal damage in Alzheimer disease (AD) and possibly in other chronic neurodegenerative diseases as well. The evidence is of two types: (i) the identification in postmortem brains of $\mathrm{AD}$ and other neurological cases of activated immune system cells (microglia/macrophages), as well as a large variety of proteins characteristic of inflammatory reactions (1); and (ii) some twenty epidemiological studies which generally indicate that antiinflammatory agents inhibit

\footnotetext{
* To whom correspondence should be addressed: Kinsmen Laboratory of Neurological Research, University of British Columbia, 2255 Wesbrook Mall, Vancouver, British Columbia, Canada, V6T 1 Z3
}

the onset and slow the progress of AD. Both subjects have been extensively reviewed $(1,2)$.

\section{CHARACTERISTIC PATHOLOGY OF ALZHEIMER DISEASE}

The characteristic pathology of $\mathrm{AD}$ remains that described by Alois Alzheimer in 1906 - namely the senile plaques and neurofibrillary tangles. A major constituent of the extracellular senile plaques is betaamyloid $(\mathrm{A} \beta)$, an insoluble peptide derived from abnormal cleavage of amyloid precursor protein (APP). The neurofibrillary tangles represent insoluble fibrous material accumulating within neurons and consist mainly of an abnormally phosphorylated form of tau. APP is a normal neuronal constituent of unknown function, while tau is a microtubule associated protein. Both amyloid and tau are foci of research on possible causative factors in AD (reviewed in (3) and (4)). Research on the former is spurred by the identification of mutations in the APP gene in some families with AD (4). Research on tau is encouraged by reports that the density of tangles correlates better with the dementia of AD than does the appearance of senile plaques (e.g., (5)).

The plaques and tangles of $\mathrm{AD}$ are confined largely to the cortical regions of brain and show clear-cut regional differences within the cortex. The entorhinal cortex and hippocampus are affected early and severely, followed by the medial temporal, parietal and frontal cortices. The motor and visual cortices show little involvement until very advanced stages $(5,6)$. The early involvement of the entorhinal cortex, hippocampus and association areas explains the loss of recent memory as the prominent early symptom.

THE INFLAMMATORY REACTION OF BRAIN

Markers of inflammation provide important clues to 
Table 1. Some molecules reported elevated in association with AD lesions (reviewed in (1)).

Acute phase reactants

Cathepsins and Cystatins

Complement proteins

Complement inhibitors

Cytokines

Growth factors

Heat shock proteins

Proteoglycans

Substrates for lipoprotein receptors

Thrombin and plasmin systems

Other amyloid P, C-reactive protein, $\alpha 1$-antichymotrypsin, $\alpha 1$-antitrypsin, $\alpha 2$-macroglobulin neuronalpentraxin

cathepsins B,D,E,H,1 and S, cystatins A and C, tissue inhibitor of metalloproteinase

C1 through $\mathrm{C} 9$

$\mathrm{C} 1$ inhibitor, $\mathrm{C} 4$-binding protein, vitronectin, clusterin, protectin

interleukin-1 $\beta$, interleukin -6 , tumor necrosis factor $\alpha$

basic fibroblast growth factor, transforming growth factor $\beta$-1, epidermal growth factor, midkine heat shock protein-72, heat shock protein HSP-27

chondroitin sulfate proteoglycan, heparan sulfate proteoglycan, dermatin sulfate, proteoglycan apolipoprotein E, low density lipoprotein receptor-related protein, lipoprotein lipase, lactoferrin thrombin, tissue factor, Hageman factor, tissue plasminogen activator, urokinase, plasminogen activator

intercellular adhesion molecule-1, chromogranin A, nuclear factor-kappa B, synuclein immunological activity since any immune response involves inflammation. Inflammation is a complex process which commences with a primary reaction in tissue. This is typically followed by a secondary reaction, which involves supplementation by blood borne elements. Historic confusion about the definition of inflammation exists, since the so-called cardinal signs, of heat, redness, swelling and pain, are due to this secondary reaction. It was the great Metchnikoff, in his classic Comparative Pathology of Inflammation (7), who provided insight into the primary process. By studying simple organisms like the starfish, he showed

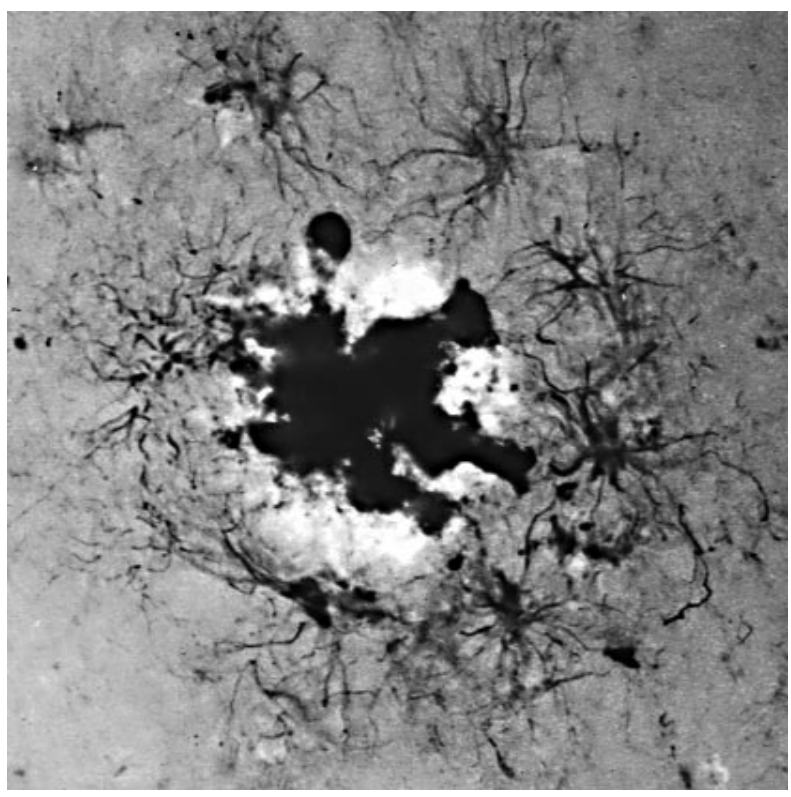

Figure 1. Triple staining of a section of Alzheimer disease cortex with antibodies for HLA-DR (staining reactive microglia; darkest color, purple on original slide) and glial fibrillary acidic protein (GFAP; lighter dark color, brown on original slide) and with thioflavin $S$ which colors amyloid beta-protein white. The amyloid deposit is the insoluble irritant, the HLA-DR-positive reactive microglia are the phagocytes attempting to remove it, and the encircling, GFAPpositive astrocytes wall off the inflamed area, marking the border between healthy and inflamed tissue. that mesodermal cells, which he named phagocytes, were the ones to act against specific irritants. Invasion of tissue by leukocytes and serum was a secondary process that was well developed in vertebrates.

For brain, it is vital that a separation of primary from secondary inflammatory processes be maintained. It relies on the blood brain barrier to protect it from the lethal consequences of typical inflammatory swelling. The capillaries are highly specialized, maintaining tight junctions to exclude molecules with osmotic potential, while still permitting leukocytes to enter when appropriate signals indicate they are required. The brain is thus a model for distinguishing between local and systemic inflammatory responses. However, since it has no pain fibers to register irritation within its matrix, the inflammation may be an unnoticed process.

In $\mathrm{AD}$ and a number of other neurodegenerative diseases, the lesions are marked by opsonizing components of the classical complement pathway and are invaded by activated microglia (Figure 1). None of these inflammatory signs are seen in the brains of persons dying without neurological disease (1). The amyloid deposits in AD are also associated with many other extracellular molecules. They include complement proteins, complement inhibitors, acute phase reactants, growth factors, heat shock proteins, proteoglycans, lipoproteins, cathepsins, cystatins, coagulation factors, proteases, protease inhibitors, integrin adhesion molecules and other miscellaneous proteins (Table 1) (1). The accumulation of these extracellular molecules is probably representative of what occurs around any chronic inflammatory lesion where attempts to resolve the irritant are unsuccessful. A situation occurs in which inflammatory forces compete with healing, or antiinflammatory forces. A stalemate results. In the case of brain, neurons and their processes may be the victims of toxic inflammatory products generated to overcome the counter-effects of protease inhibitors and other antiinflammatory products. 


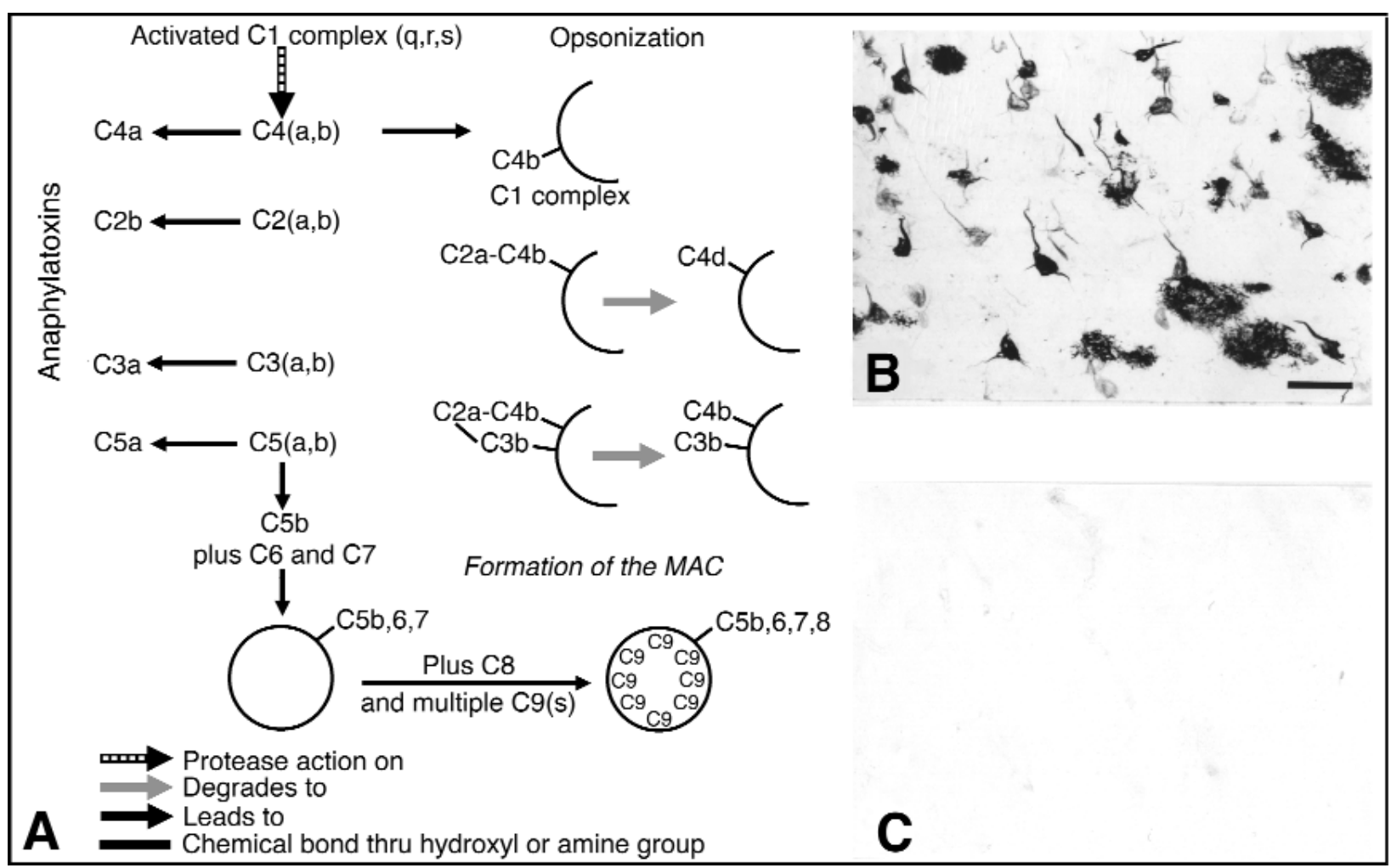

Figure 2. A diagram of the classical complement cascade (A), and staining of sections from the temporal cortex of a patient with AD (B) and an age-matched control (C) with an antibody to $\mathrm{C} 4 \mathrm{~d}$. The plaques and tangles of $\mathrm{AD}$ are intensely stained.

Studies of cultured human astrocytes and microglia, obtained from postmortem brain, have established that nearly all of the inflammatory proteins seen in $\mathrm{AD}$ tissue are produced by microglia, astrocytes or both (1). The appearance of reactive astrocytes, along with reactive microglia, in association with the lesions of $\mathrm{AD}$ and some other neurodegenerative diseases, is well documented (Figure 1) (8). Human neurons also produce many inflammatory proteins and their inhibitors, creating complex interactions. Thus, the brain clearly has its own immune response system which involves neurons as well as glia. However, microglia are the pivotal cells.

Microglia are the brain's representatives of the monocyte phagocytic system. In normal adult brain, microglia generally show resting morphology. Injuries of various types in animal models lead to rapid activation. In vitro, microglia can be activated by numerous materials including complement proteins (9), $\mathrm{A} \beta$ (10) and chromogranin A (11), all of which are found associated with AD lesions (Table 1). Activated microglia will undergo respiratory burst activity. This property is characteristic of cells of the monocyte phagocytic system, and of circulating neutrophils. All these professional phagocytes possess, in common, a system which can generate prodigious quantities of superoxide anions from oxygen molecules. Hydroxyl radicals, singlet oxygen species and hydrogen peroxide are downstream products. The system is one of the main methods by which phagocytes kill invading organisms and tumor cells. Activated microglia also secrete much of the potentially neurotoxic glutamate (12), and possibly other unidentified toxins. The purpose, of course, is phagocytosis, and some might argue that the activated microglia in $\mathrm{AD}$ are there only to clean up the debris. But the oxidative stress and other potential toxins may not distinguish friend from foe, so the possibility must be kept in mind that microglia may contribute substantially to autotoxic tissue damage. That this may well be true is supported by evidence from studies on mixed neuronal/microglial cultures indicating that activated microglia secrete products toxic to neurons (13-16).

Proteins of the classical complement cascade (Figure 2) may also be of particular importance. This cascade produces not only proteins which mark structures for opsonization but also anaphyloxins which spur on the immune system and, if chronically activated, the membrane attack complex (the MAC, C5b-9). Complement has traditionally been thought of as a blood-borne system activated by antigen-antibody complexes. However, in situ hybridization studies and 
RISK FACTORS

Genes, Age, Environment, Others

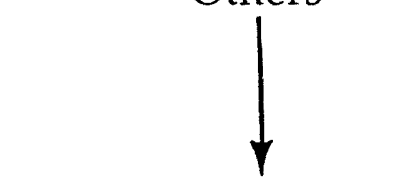

PATHOLOGY

Senile plaques, Neurofibrillary tangles, Lewy bodies, etc

\section{AUTOTOXIC LOOP}

Autotoxic Products

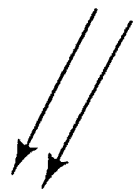

Inflammatory Cytokines
Microglial Activation
Complement Activation

Figure 3. Autotoxic loop hypothesized as responsible for much of the neuronal death in Alzheimer disease and possibly other neurodegenerative disorders.

immunohistochemical studies have shown that both the mRNAs for all the complement proteins, and the proteins themselves, are located prominently in neurons, especially in the cortical pyramidal neurons which are severely affected in AD $(17,18)$. Both mRNAs and proteins are upregulated in these neurons in $\mathrm{AD}(17,18)$. Whether complement-producing neurons are particularly vulnerable to immune system attack remains to be explored.

Complement proteins might, however, contribute to neuronal death in a number of ways. Opsonizing components of the complement cascade may mistakenly mark host tissue for opsonization. The most compelling immunohistochemical evidence for autodestruction of neuronal processes by complement components, however, is the presence of the MAC on dystrophic neurites in senile plaques. Such dystrophic neurites appear in classical senile plaques but not diffuse amyloid deposits. The MAC inserts into the membranes of intact cells, causing lysis and death of the cell. The MAC is intended to destroy foreign cells and viruses, but host cells are at significant risk of bystander lysis. There has been some controversy as to the role of the MAC in AD pathology due to one group who reported that the MAC is not present on $A D$ pathological structures (19). However, the current authors' results $(20,21)$, and those of others (22), have conclusively demonstrated its presence by immunohistochemistry and Western blotting.

Further evidence of a chronic inflammatory reaction in $\mathrm{AD}$, as well as in some other neurodegenerative diseases, is provided by reports of increased levels of inflammatory cytokines and their receptors, such as interleukin-1 $\beta$ (IL-1 $\beta$ ), IL-6, the type B IL-8 receptor and the receptor for colony stimulating factor-1 (CSF-1) in brain tissue and/or CSF (reviewed in (23)). A number of interactions in vitro between cytokines and components that have been identified in AD senile plaques have been reported, suggesting a further way in which a vicious cycle might be generated (also reviewed in (23)). For example, $\mathrm{A} \beta$ bas been found to potentiate the secretion of IL- 6 and IL- 8 by IL- $1 \beta$ activated astrocytoma cells, and of IL- 8 by monocytes. In turn, IL-1, IL-2, IL-6 and granulocyte/macrophagecolony stimulating factor (GM-CSF) have all been reported to stimulate expression of the APP gene, which codes for the amyloid precursor protein, in neuronal cultures. IL-1 has also been found to increase the secretion of proteoglycans such as heparin sulfate by neuroblastoma cells and to induce expression of $\alpha$ antichymotrypsin, thromboplastin, complement protein C3 and apolipoprotein E by appropriate cell lines. Thus, cytokines may stimulate secretion of a number of the proteins found in senile plaques.

The enhanced expression of nuclear factor-kappa B $(\mathrm{NF}-\mathrm{\kappa B})$ in neurons in AD brain (24) is also consistent with an inflammatory reaction, since $\mathrm{NF}-\kappa \mathrm{B}$ is presently regarded as a central regulator of inflammatory, immune and acute phase reactions (25).

\section{AUTOTOXIC HYPOTHESIS}

The current authors have proposed the autotoxic loop hypothesis, which suggests that much of the neuronal damage in $\mathrm{AD}$, and possibly other neurodegenerative diseases, is due to a locally self-sustaining chronic inflammatory reaction (1). The autotoxic loop model (Figure 3) is compatible with the clinically observed change in $\mathrm{AD}$ from an initial slow progression to a rapid decline $(26,27)$. Various predisposing factors, such as genetic make-up, age, and possibly environmental toxins, contribute to the development of initial lesions. In the case of $\mathrm{AD}$, these are the neurofibrillary tangles 


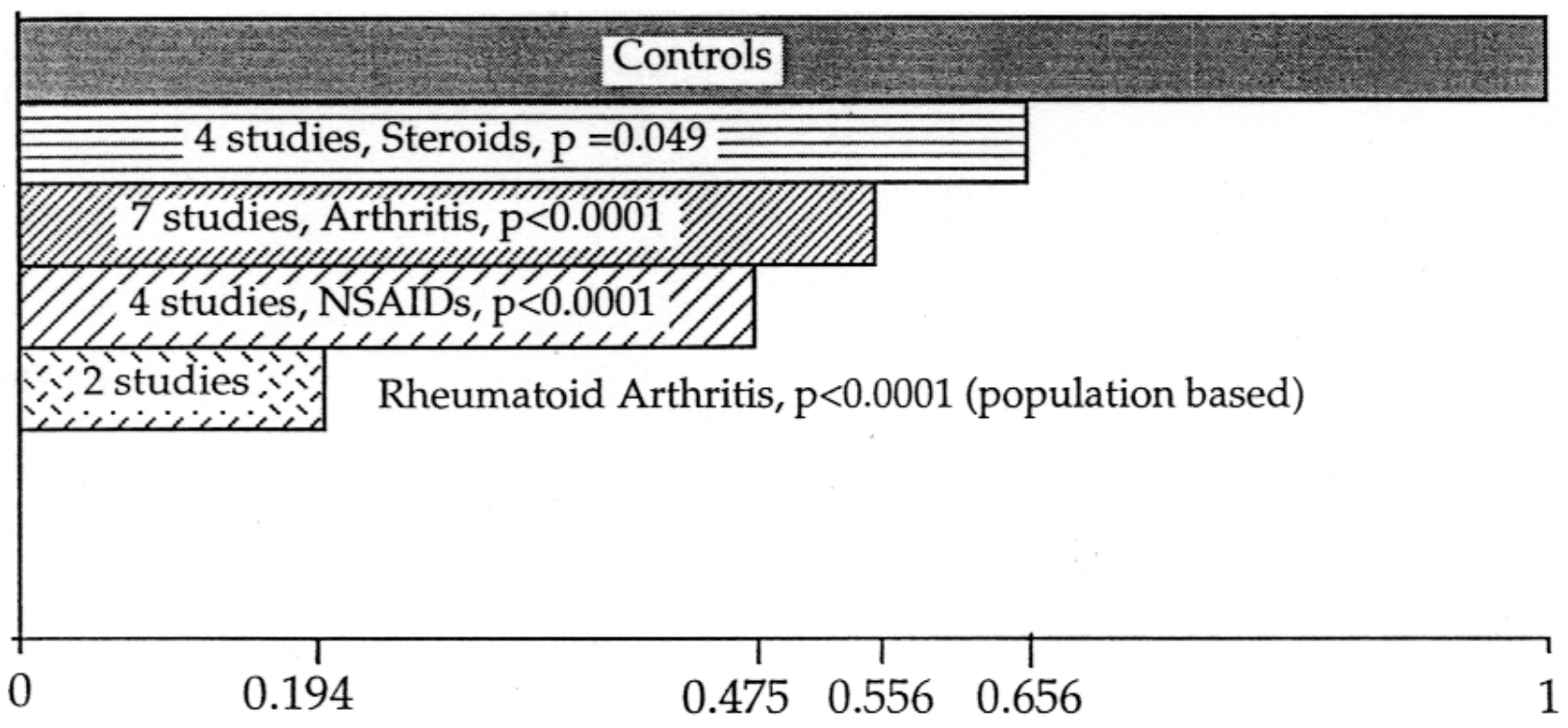

Figure 4. Results of a meta-analysis of homogeneous studies on arthritis or use of antiinflammatory drugs as a risk factor for Alzheimer disease. The calculated odds ratio and $\mathrm{p}$ value are shown for each group. All groups involved case controlled studies except where population based is specified. This graph includes the data from the paper by Stewart et al. (46) as well as the 17 studies reported in our published meta-analysis (2).

and senile plaques. Presumably these lesions themselves lead to neuronal death, but the process may not be an aggressive one so that, without inflammatory amplification, the progression may be slow. Although the lesions stimulate an inflammatory response, this may initially be below the threshold which will contribute significantly to further neuronal damage. However, it eventually reaches a level where it does cause neuronal death, and that process itself generates still more lesions, which further exacerbate the inflammatory response. This positive feedback loop produces the rapid decline that is observed clinically.

This is not an autoimmune disease in the classical sense of the development of antibodies to host tissue. No reliable evidence exists for such antibodies in AD (1). In experimental models of brain injury, activation of the complement pathway and appearance of reactive microglia occur much too rapidly to allow for the production of autoantibodies $(28,29)$ and it is now clear that the complement cascade can be activated in the absence of antibodies. Several of the proteins occurring in association with the lesions in $\mathrm{AD}$ have been found to activate the complement cascade in vitro. These include serum amyloid $\mathrm{P}$, the Hageman factor, Creactive protein and $A \beta$ itself (1). One interesting point is that some of these materials activate complement by binding to the collagen tail of $\mathrm{C} 1 \mathrm{q}$, rather than to the globular head, as do antibody/antigen complexes (30, 31). This has therapeutic implications, since specific blockers of binding to the collagen tail might inhibit non-immune activation of complement, while preserving antibody activation.
Several groups, using different types of neurons and neuronal cell lines (32-35), have even shown that such cells can activate complement in the absence of specific antibodies. This is quite a remarkable property, as it renders the cells potentially vulnerable to self-inflicted damage. Such damage is normally prevented by homologous restriction factors, especially protectin (also known as CD59). The current authors have shown the upregulation of such restriction factors as protectin, vitronectin and clusterin near senile plaques in $\mathrm{AD}$ tissue, suggesting an attempt at defense (36-38). The present authors have also shown that $\mathrm{C} 1$ inhibitor appears on dystrophic neurites associated with classical senile plaques in AD tissue (39), thus having a distribution similar to that of the MAC. Damage to neurons may depend upon the relative balance between complement inhibitors and the strength of complement attack.

The autotoxic loop concept is not one that is limited to $\mathrm{AD}$, or even to other degenerative brain disorders. It might apply to a diverse spectrum of chronic inflammatory diseases where evidence of systemic involvement is minimal or absent (40). Markers of peripheral immune system participation, such as specific antibodies or cloned T-cell subsets, need not be present. All that is required is the presence of a positive feedback loop as shown in Figure 3. Once the inflammatory response reaches the threshold necessary to produce autodestruction of viable host cells, the reaction can be sustained by further production of inflammatory stimulants. 


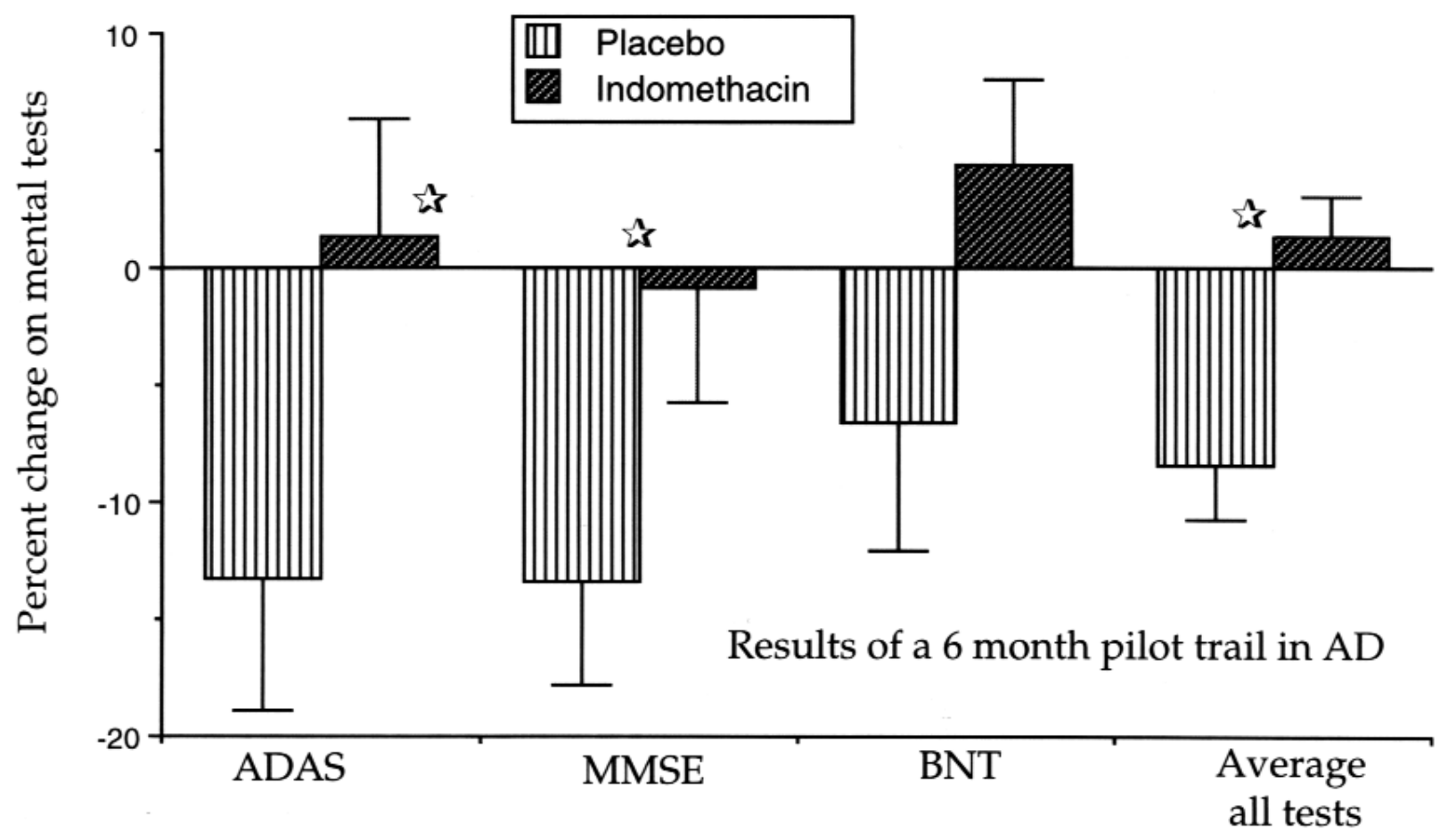

Figure 5. Average (mean \pm 1 S.E.) changes over 6 months as percent of base line on mental status tests shown by AD patients on indomethacin as compared to those on placebo. The data come from the study of Rogers et al. (44).

POSSIBLE THERAPEUTIC USE OF ANTIINFLAMMATORY AGENTS

The accumulating evidence of a chronic innate immune reaction in $\mathrm{AD}$ brain led to the suggestion that chronic treatment with antiinflammatory agents might slow the progress of $\mathrm{AD}$ or retard its onset (41). It is important to realize that this therapeutic approach to $\mathrm{AD}$ is very different from attempts to replace the lost cholinergic activity or stimulate the cortical glucose metabolism. Such pharmacological approaches do not attempt to slow the very rapid destruction of cortical neurons which is almost certainly the main cause of the mental deterioration. The suggestion is that antiinflammatory treatment may inhibit such neuronal death. There are now twenty published epidemiological studies and one reported clinical trial which generally support this possibility. Many of these studies are relevant because they considered arthritis, a major indication for the use of antiinflammatory drugs, or antiinflammatory drugs themselves, as risk factors for AD. In some of the studies, other diseases or drugs were considered as possible risk factors, but only arthritis and antiinflammatory drug use have generally shown negative associations with $\mathrm{AD}$.

The outcome of a risk factor analysis is often expressed as an odds ratio. This is a commonly used statistic for estimation of the relative risk of developing the disorder if there is exposure to a particular factor. It measures the odds that a person in an exposed group will develop $\mathrm{AD}$, relative to the corresponding odds for a person in a control, or non-exposed group. A negative risk, corresponding to a protective factor, yields an odds ratio less than one, while a positive risk factor is associated with an odds ratio greater than one. Estimating overall odds ratios using data from multiple reports requires that the studies be similar in design. The current authors therefore grouped the seventeen studies published at the time of the analysis according to the risk factor employed and into case control or population based. For groups which passed a test of homogeneity (similarity in design), a meta-analysis was done to determine the overall risk factor and its significance (2). The results are shown in Figure 4, and clearly support the hypothesis that the use of antiinflammatory agents, particularly the non-steroidal antiinflammatory drugs (NSAIDs), will decrease the risk of $\mathrm{AD}$.

Two additional papers $(42,43)$ have reported retrospective studies of clinical charts which indicate that $\mathrm{AD}$ patients taking NSAIDs for other purposes have a slower progression of the disease. The only clinical trial of the effects of an NSAID for the treatment of $\mathrm{AD}$, however, was a small, double blind, placebo controlled study in which indomethacin appeared to arrest the progression of the disease for the 6 month period of the trial (Figure 5) (44). 
Taken together, these data suggest that the disciplined use of an effective NSAID should be able to cut the prevalence of Alzheimer disease at least in half. Reluctance to treat geriatric cases with NSAIDs exists in the medical community. This is based upon the known side effects of these drugs, the most dangerous of which is inducing gastrointestinal ulceration and bleeding. Although agents like misoprostol offer some protection against this major side effect, it remains a concern. What needs to be balanced against such risks are the rewards that epidemiological studies suggest might result from NSAID use. These are economic as well as humanitarian. For example, the annual cost of caring for demented patients in Canada with its population of about 25 million has been estimated at $\$ 3.9$ billion (after deducting costs associated with the medical care of age matched non-demented controls) (45). One can readily calculate the pharmacoeconomic benefits of reducing the prevalence even by as little as half. And the benefit in alleviation of human suffering is obvious but incalculable. Thus, any treatment which may inhibit the onset and slow the progress of $\mathrm{AD}$ seems worth thorough investigation.

\section{ACKNOWLEDGMENTS}

Our research on Alzheimer disease is supported by grants from the Alzheimer Society of B.C., the Jack Brown and Family A.D. Research Fund and donations from individual British Columbians.

\section{REFERENCES}

1. McGeer PL, McGeer EG. The inflammatory system of brain: implications for therapy of Alzheimer and other neurodegenerative disorders. Brain Research Review 21(2):195218; 1995.

2. McGeer PL, Schulzer M, McGeer EG. Arthritis and antiinflammatory agents as negative risk factors for Alzheimer disease: A review of seventeen epidemiological studies. Neurology 47(2):425-432; 1996.

3. Selkoe DJ. Cell biology of the beta-amyloid protein and the genetics of Alzheimer's disease. Cold Spring Harbor Symposia on Quantitative Biology 61:587-596; 1996.

4. Iqbal K, Grundke-Iqbal I. Molecular mechanism of Alzheimer's neurofibrillary degeneration and therapeutic intervention. Annals of the New York Academy of Sciences 777:132-138; 1996.

5. Braak H, Braak E. Evolution of the neuropathology of Alzheimer's disease. Acta Neurologica Scandinavica Supplementum 165:3-12; 1996.

6. Brun A, Englund E. Regional pattern of degeneration in Alzheimer's disease: neuronal loss and histopathological grading. Histopathology 5(5):549-564; 1981.

7. Metchnikoff E. Leaons sur la pathologie comparée de l'inflammation. Paris: Masson; 1892.

8. Pike CJ, Cummings BJ, Monzavi R, Cotman CW. Beta-amyloid induced changes in cultured astrocytes parallel reactive astrocytosis associated with senile plaques in Alzheimer's disease. Neuroscience 63(2):517-531; 1994.
9. Klegeris A, McGeer PL. Rat brain microglia and peritoneal macrophages show similar responses to respiratory burst stimulants. Journal of Neuroimmunology 53(1):83-90;1994.

10. Klegeris A, Walker DG, McGeer PL. Activation of macrophages by Alzheimer $\beta$ amyloid peptide. Biochemical and Biophysical Research Communications 199(2):984-991; 1994.

11. Taupenot L, Ciesielski-Traska J, et al. Chromogranin A triggers a phenotypic transformation and the generation of nitric oxide in brain microglial cells. Neuroscience 72(2): 377-389; 1996.

12. Klegeris A, McGeer PL. $\beta$-Amyloid protein enhances macrophage production of oxygen free radicals and glutamate. Journal of Neuroscience Research 49(2): 229-2357; 1997.

13. McMillian MK, Vainio PJ, Tuominen RK. Role of protein kinase $\mathrm{C}$ in microglia-induced neurotoxicity in mesencephalic cultures. Journal of Neuropathology and Experimental Neurology 56(3):301-307; 1997.

14. Giulian D, Haverkamp LJ, Yu JH, et al. Specific domains of $\beta$ amyloid from Alzheimer plaque elicit neuronal killing in human microglia. Journal of Neuroscience 16(19):6021-6037; 1996.

15. Roher AE, Chaney MO, Kuo YM, et al. Morophology and toxicity of A-beta (1-42) derived from neuritic and vascular amyloid deposits of Alzheimer's disease. Journal of Biological Chemistry 271(34):20631-20635;1996.

16. Giulian D, Vaca K, Corpuz M. Brain glia release factors with opposing actions upon neuronal survival. Journal of Neuroscience 13(1):29-37; 1993.

17. Shen Y, Li R, McGeer EG, McGeer PL. Neuronal expression of mRNAs for complement proteins of the classical pathway in Alzheimer brain. Brain Research 769(2):391-395; 1997.

18. Terai K, McGeer EG, McGeer PL. Neurons express proteins of the classical complement pathway in Alzheimer disease. Brain Research 769(2):385-390; 1997.

19. Veerhuis R, van der Valk P, Janssen I, et al. Complement activation in amyloid plaques in Alzheimer's disease brains does not proceed further than C3. Virchows Archiv 426(6): 603-610; 1995.

20. McGeer PL, McGeer EG. Kawamata T, et al. Reactions of the immune system in chronic degenerative neurological diseases. Canadian Journal of Neurological Sciences 18(3 Suppl):376379; 1991.

21. Itagaki S, Akiyama $\mathrm{H}$, Saito $\mathrm{H}$, et al. Ultrastructural localization of complement membrane attack complex (MAC)-like immunoreactivity in brains of patients with Alzheimer's disease. Brain Research 645(1-2):78-84; 1994.

22. Webster S, Lue L-F, Brachova L, et al. Molecular and cellular characterization of the membrane attack complex, C5b-9, in Alzheimer's disease. Neurobiology of Aging 18(4): 415-421; 1997.

23. McGeer EG, McGeer PL. Inflammatory cytokines in the CNS. CNS Drugs 7: 214-228; 1997.

24. Terai K, Matsuo A, McGeer PL. Enhancement of immunoreactivity for NF- $\mathrm{KB}$ in the hippocampal formation and cerebral cortex of Alzheimer's disease. Brain Research 735(1):159-168; 1996.

25. Conner EM, Grisham MB. Inflammation, free radicals and antioxidants. Nutrition 12(4): 274-277; 1996.

26. Helmes E, Merskey H, Fox H, et al. Patterns of deterioration in senile dementia of the Alzheimer type. Archives of Neurology 52(3):306-310; 1995.

27. Ohm TG, Muller H, Braak H, et al. Close-meshed prevalence rates of different stages as a tool to uncover the rate of Alzheimer's disease-related neurofibrillary changes, Neuroscience 64(1):209-217; 1995.

28. Akiyama H, Tooyama I, Kondo H, et al. Early response of brain resident microglia to kainic acid-induced hippocampal lesions. 
Brain Research 635(1-2):257-268; 1994.

29. Pasinetti GM, Johnson SA, Rozovsky I, et al. Complement $\mathrm{C} 1 \mathrm{qB}$ and $\mathrm{C} 4 \mathrm{mRNAs}$ responses to lesioning in rat brain. Experimental Neurology 118(2):117-125; 1992.

30. Jiang H, Burdick D, Glabe GG, et al. $\beta$-Amyloid activates complement by binding to a specific region of the collagen-like domain of the $\mathrm{C} 1 \mathrm{q}$ A chain. Journal of Immunology 152(10):5050-5059; 1994.

31. Rogers J, Cooper NR, Webster S, et al. Complement activation by $\beta$-amyloid in Alzheimer disease. Proceedings of the National Academy of Science USA 89(21):10016-10020; 1992.

32. Gasque $\mathrm{P}$, Thomas A, Fontaine M, et al. Complement activation on human neuroblastoma cell lines in vitro: route of activation and expression of functional complement regulatory proteins. Journal of Neuroimmunology 66(1-2):29-40; 1996.

33. Shen Y, Halperin JA, Lee CM. Complement-mediated neurotoxicity is regulated by homologous restriction. Brain Research 671(2):282-292; 1995.

34. Pasinetti GM. Inflammatory mechanisms in neurodegeneration in Alzheimer's disease: the role of the complement system. Neurobiology of Aging 17(5):707-716; 1996.

35. Agoropoulou C, Wing MG, Wood A. CD59 expression and complement susceptibility of human neuronal cell line (NTera2). Neuroreport 7(5):997-1004; 1996.

36. McGeer PL, Walker DG, Akiyama H, et al. Detection of the membrane inhibitor of reactive lysis (CD59) in diseased neurons of Alzheimer brain. Brain Research 544(2):315-319; 1991.

37. McGeer PL, Kawamata T, Walker DG. Distribution of clusterin in Alzheimer brain tissue. Brain Research 579(2):337-341;
1992.

38. Akiyama H, Kawamata $\mathrm{T}$, Dedhar $\mathrm{S}$, et al. Immunohistochemcial localization of vitronectin, its receptor and beta-3 integrin in Alzheimer brain tissue. Journal of Neuroimmunology 32(1):19-28; 1991.

39. Walker DG, Yasuhara O, Patston PA, et al. Complement C1 inhibitor is produced by brain tissue and is cleaved in Alzheimer disease. Brain Research 675(1-2):75-82; 1995.

40. Laskin DL, Pendino KJ. Macrophages and inflammatory mediators in tissue injury. Annual Review of Pharmacology and Toxicology 35:655-677; 1995.

41. McGeer PL, Rogers J. Anti-inflammatory agents as a therapeutic approach to Alzheimer's disease. Neurology 42(2):447-449; 1992.

42. Rich JB, Rasmusson DX, Folstein MF, et al. Nonsteroidal antiinflammatory drugs in Alzheimer's disease. Neurology 45(1):51-55; 1995.

43. Doraiswamy PM, Krishen A, Stallone F, et al. (1996) NSAIDs and cognition in Alzheimer's disease. Neurology 46(4):1194; 1996.

44. Rogers J, Kirby LC, Hempelman SR, et al. Clinical trial of indomethacin in Alzheimer's disease. Neurology 43(8): 1609$1611 ; 1993$.

45. Ostbye T, Crosse E. Net economic costs of dementia in Canada. Canadian Medical Association Journal 151(10):1457-1464; 1994.

46. Stewart WF, Kawas C, Corrada M, et al. Risk of Alzheimer's disease and duration of NSAID use. Neurology 48(3): 626-632; 1997.

Edith G. McGeer, Ph.D., and Patrick L. McGeer, M.D., are professor emeriti at the University of British Columbia (Vancouver, British Columbia, Canada) where they have been working in the field of neuroscience since 1954 (before the first neurotransmitter was definitely identified in brain). Their work for the past 10 years has been mainly on the role of the immune system in neurodegenerative diseases, with particular emphasis on Alzheimer disease. 